\title{
COVID-19 in a Leukemic Child
}

\author{
Muhd Muhd Helmi ${ }^{1}$, Norsarwany Mohamad ${ }^{2}$, Fahisham Taib², Ariffin Nasir ${ }^{2}$, and Nik \\ Khairulddin NBik Yusoff ${ }^{3}$ \\ ${ }^{1}$ International Islamic University Malaysia - Kuantan Campus \\ ${ }^{2}$ Universiti Sains Malaysia Pusat Pengajian Sains Perubatan \\ ${ }^{3}$ Hospital Raja Perempuan Zainab II
}

September 28, 2020

\begin{abstract}
The newly emerged SARS-CoV-2 Betacoronavirus strain has infected more than one million people worldwide. The COVID-19 infection in children was reported to be less severe compared to adults. At this point, there were limited data available to describe the effect of COVID-19 infection in immunocompromised children. We reported a case of SARS-CoV-2 infection in an immunocompromised child with a delayed virus clearance. The report signifies the mild severity of SARS-CoV-2 infection in immunocompromised children
\end{abstract}

Main text word count: 640

Keywords: COVID-19, SARS-CoV-2, Immunocompromised

\begin{tabular}{ll}
\hline Abbreviation & Full term \\
\hline HCoV & Human Coronavirus \\
rT-PCR & Real-time Polymerase Chain reaction \\
\hline
\end{tabular}

\section{TITLE}

Delayed Viral Clearance in an Immunocompromised Child with Covid-19 Infection

\begin{abstract}
The newly emerged SARS-CoV-2 Betacoronavirus strain has infected more than one million people worldwide. The COVID-19 infection in children was reported to be less severe compared to adults. At this point, there were limited data available to describe the effect of COVID-19 infection in immunocompromised children. We reported a case of SARS-CoV-2 infection in an immunocompromised child with a delayed virus clearance. The report signifies the mild severity of SARS-CoV-2 infection in immunocompromised children.
\end{abstract}

\section{TEXT}

\section{Introduction}

Globally, approximately $2 \%$ of those affected by COVID-19 were children, with less severe disease course compared to adult. ${ }^{1,2}$ Global data on the effect of COVID-19 infection to immunocompromised children were scarce. This scarcity is possibly due to the small number of positive cases and the majority of asymptomatic children were left unscreened. We report a case of a leukemic who contracted COVID-19 infection presented with delayed viral clearance. 


\section{Results}

We reported a 27-month-old boy, known case of B-cell Acute Lymphoblastic Leukemia who was commenced on chemotherapy in late 2019. He was in remission but had to postpone his chemotherapy due to postinduction chemotherapy neutropenia. He stayed with his mother, two siblings and their extended family members. He was screened for COVID-19 infection after revealing that he had contact with his two extended family members who were positive for COVID-19. They had travelled interstate to a wedding function (nine days before his rescheduled chemotherapy) and subsequently developed a cough and sore throat without fever. Social distancing was not practised among the household members. He was transferred to a COVID19 designated hospital for quarantine and observation but remained asymptomatic during his 16 days of hospital stay.

His chest radiograph showed minimal consolidative changes. Full blood counts on admission showed white cell count of 12x109/L, neutrophil count of 4.9x109/L, lymphocytes count of 4.24x109/L, and platelet counts of 337x109/L. Renal and liver function test, were within the normal value for age. C-reactive protein was twice negative. SARS-CoV-2 real-time Polymerase Chain reaction (rT-PCR) were repeated at day 10th, 13 th and 16th of admission. His last naso- and oropharyngeal swab at day 16 were negative, and he was discharged well to continue for another one week of home quarantine. The patient completed his home quarantine period asymptomatically, and chemotherapy was commenced after that.

\section{Discussion}

In the epidemiological study from China, ninety per cent of the SARS-CoV-2 infected children had mild to moderate symptoms. ${ }^{3}$ Younger children, less than 5 -year-old, were more likely to develop a complication. ${ }^{3}$ Majority of symptomatic children with COVID-19 presented with upper respiratory symptomatology. ${ }^{1}$ There was a lack of data on COVID-19 infection in immunocompromised children. However, the outcome can be predicted based on the effect of other Human Coronavirus (HCoV) infection to immunocompromised children. In a retrospective study, both of the immunocompromised and immunocompetent children with $\mathrm{HCoV}$ infection presented similarly, mainly with fever, cough and sore throat. ${ }^{4}$ The severity was identical even with different strains of HCoV. ${ }^{1,4}$ However, immunocompromised children were more likely to develop severe pneumonia compared to immunocompetent children. ${ }^{4}$

Morbidity and mortality in the patients with COVID-19 are thought to be due to hyper-inflammatory state such as cytokine storm syndrome. ${ }^{5}$ It is thought that immature and poor functioning ACE-2 receptor which reduced the SARS-CoV-2 virus affinity to the receptor, higher level of antibody against respiratory viruses, the cross protection of childhood immunisation program, and the lack of coronavirus-specific memory cells have led to reduce direct cell-mediated attack and inflammation on alveoli and other organs. ${ }^{3,6}$ This exaggerated innate immune response was not seen in the immunocompromised children. ${ }^{2,7}$ During the SARSCoV outbreak in 2002/2003, children with organ transplant had good outcome. ${ }^{7}$ Similarly, there was no documented severe COVID-19 pneumonia among paediatric liver transplant patients in Italy. ${ }^{7}$

In contrast to the reported case of COVID-19 positive leukemic child by Chen et al., our patient was asymptomatic throughout the disease course. The reported child developed a high-grade fever which progressed into pneumonia requiring intensive care unit admission. ${ }^{8}$ The severe neutropenia and concomitant influenza A infection might have contributed to more severe disease in the reported child. ${ }^{8}$

In conclusion, this report and other previous cases of COVID-19 in immunocompromised children revealed that the infection is mild. The severity may however be worse with concomitant other respiratory virus infection. Further evidence on the pathogenesis and outcome of SARS-CoV-2 infection on immunocompromised children are required before any conclusion can be made.

\section{Conflicts of Interest Statement}

None of the author have conflicts of interest to be declared

\section{Acknowledgement}


All authors contributed equally. We would like to acknowledge the Director-General of Health, Ministry of Health Malaysia, Datuk Dr Noor Hisham Abdullah, the Director of Hospital Universiti Sains Malaysia, Profesor Dato' Dr Ahmad Sukari Bin Halim, and Dato' Dr Hjh Selasawati bt. Hj. Ghazali, the Director of Hospital Raja Perempuan Zainab II, for the support they have shown towards the publication of this report. To Dr Alia Rashid and Dr Ummuatiyah, medical officers at Severe Acute Respiratory Infection ward, Hospital Raja Perempuan Zainab II for the information that they have provided. To medical and house officers of paediatric haematooncology ward, Hospital University Sains Malaysia. To Mrs Nurul Azurah Mohd Roni and Mrs Noraida Hassan, the two librarians of Universiti Sains Malaysia who have assisted us on referencing and literature searching. Finally, the reported patient and his family members for the consent and cooperation they have given throughout the writing of this report.

\section{Reference}

1. Zimmermann P, Curtis N. Coronavirus Infections in Children Including COVID-19: An Overview of the Epidemiology, Clinical Features, Diagnosis, Treatment and Prevention Options in Children. The Pediatric infectious disease journal 2020;39(5):355.

2. Akcabelen YM, Yozgat AK, Parlakay AN, Yarali N. COVID-19 in a child with severe aplastic anemia. Pediatric blood \& cancer 2020;67(8):e28443.

3. Dong Y, Mo X, Hu Y, Qi X, Jiang F, Jiang Z, et al. Epidemiological characteristics of 2143 pediatric patients with 2019 coronavirus disease in China. J Emerg Med 2020;58(4):712-713.

4. Ogimi C, Englund JA, Bradford MC, Qin X, Boeckh M, Waghmare A. Characteristics and outcomes of coronavirus infection in children: the role of viral factors and an immunocompromised state. Journal of the Pediatric Infectious Diseases Society. 2019;8(1):21-8.

5. Fishman JA, Grossi PA. Novel Coronavirus-19 (COVID-19) in the Immunocompromised Transplant Recipient:\# Flatteningthecurve. Am J Transplant 2020;20:1765-1767.

6. Okyay RA, Sahin AR, Aguinada RA, Tasdogan AM. Why are Children Less Affected by COVID-19? Could there be an Overlooked Bacterial Co-Infection? Pediatrics 2019;1:8.

7. D'Antiga L. Coronaviruses and immunosuppressed patients. The facts during the third epidemic. Liver Transpl 2020;26:832-834.

8. Chen Z, Xiong H, Li J, Li H, Tao F, Yang Y, et al. COVID-19 with post-chemotherapy agranulocytosis in childhood acute leukemia: a case report. Zhonghua xueyexue zazhi 2020;41:E004. 